\title{
Epidemiology of laboratory-confirmed respiratory syncytial virus infection in young children in England, 2010-2014: the importance of birth month
}

\author{
R. M. REEVES $S^{1,2,3 *}$, P. HARDELID ${ }^{1,2}$, R. GILBERT ${ }^{1,2}$, J. ELLIS $^{4}$, H. ZHAO $^{3}$, \\ M. DONATI ${ }^{5}$ AND R. PEBODY ${ }^{3}$ \\ ${ }^{1}$ Farr Institute of Health Informatics Research, London, UK \\ ${ }^{2}$ Institute of Child Health, University College London, UK \\ ${ }^{3}$ Respiratory Diseases Department, Public Health England, Colindale, London, UK \\ ${ }^{4}$ Virus Reference Department, Public Health England, Colindale, London, UK \\ ${ }^{5}$ Bristol Public Health Laboratory, Public Health England, Bristol, UK
}

Received 2 December 2015; Final revision 23 January 2016; Accepted 3 February 2016; first published online 26 February 2016

\section{SUMMARY}

The epidemiology of laboratory-confirmed respiratory syncytial virus (RSV) infections in young children has not recently been described in England, and is an essential step in identifying optimal target groups for future licensed RSV vaccines. We used two laboratory surveillance systems to examine the total number and number of positive RSV tests in children aged $<5$ years in England from 2010 to 2014. We derived odds ratios (ORs) with $95 \%$ confidence intervals (CIs) comparing children by birth month, using multivariable logistic regression models adjusted for age, season and sex. Forty-seven percent of RSV tests ( 29 851/63 827) and 57\% (7405/13 034) of positive results in children aged $<5$ years were in infants aged $<6$ months. Moreover, $38 \%$ (4982/13 034) of positive results were in infants aged $<3$ months. Infants born in September, October and November had the highest odds of a positive RSV test during their first year of life compared to infants born in January (OR 2.1, 95\% CI 1·7-2.7; OR $2 \cdot 4$,

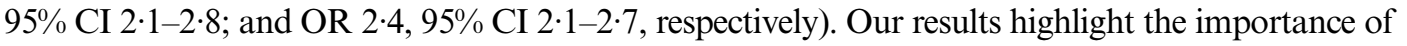
young age and birth month near the beginning of the RSV season to the risk of laboratory-confirmed RSV infection. Future control measures should consider protection for these groups.

Key words: Children, England, epidemiology, laboratory-confirmed, respiratory syncytial virus.

\section{INTRODUCTION}

Respiratory syncytial virus (RSV) is a major cause of hospitalization with lower respiratory tract infections in young children worldwide [1]. RSV infection in early life is associated with development of long-term respiratory morbidity including asthma and recurrent wheezing [1]. In developing countries, RSV is also an important

\footnotetext{
* Author for correspondence: Miss R. M. Reeves, Respiratory Diseases Department, Public Health England, 61 Colindale Avenue, London NW9 5EQ, UK.

(Email: Rachel.Reeves@phe.gov.uk)
}

cause of childhood mortality [2]. Risk factors for severe RSV infection resulting in hospitalization have been identified such as prematurity, chronic lung disease and congenital heart disease [3]. However, it has been estimated that four fifths of RSV-associated hospitalizations in infancy occur in previously healthy infants born at term, with young age a significant risk factor for severe RSV infection [4]. Consequently, RSV is a high priority for vaccine development, with several vaccines now in phase two clinical trials [5]. Potential target groups for a future RSV vaccine have been identified, including pregnant women, young infants and children aged 6-24 months, although further work is needed to 
determine the optimal age and target groups for a potential future licensed vaccine [6].

Birth in close proximity to the beginning of the RSV season has been shown to be a significant risk factor for RSV-associated hospitalization in infancy [7-11]. However, these studies rely solely on clinical diagnoses, are based outside of the UK or are of small study populations [7]. With various causative agents of upper and lower respiratory tract infections and testing only carried out in a minority of cases, relying on clinical diagnoses without laboratory confirmation of RSV may bias any observed associations between patient characteristics and severe RSV infection due to potential low specificity [12-14]. Using laboratory-confirmed RSV infection has the advantage of being highly specific, and is a starting point from which estimates of the burden of RSV in England can be calculated. The epidemiology of laboratoryconfirmed RSV infection in infants and young children in England has not recently been described [13-15].

This study uses laboratory surveillance data to examine the relationship between laboratory-confirmed RSV infection and age and birth month in children aged $<5$ years in England, to contribute to the identification of optimal target groups for any potential future licensed vaccine.

\section{METHODS}

\section{Data sources}

The Respiratory DataMart System (RDS) is a surveillance system established by Public Health England (PHE) during the 2009 influenza A(H1N1) pandemic to collect both positive and negative laboratory results for major respiratory viruses [16]. RSV test results through this system are available from 2010 onwards. Fourteen PHE and National Health Service (NHS) laboratories in England currently submit data to the RDS through automatic electronic outputs. The majority of tested samples have been collected from hospitals [16]. Respiratory samples are tested for a range of respiratory viral pathogens including RSV, influenza $\mathrm{A}$ and $\mathrm{B}$, parainfluenza, rhinovirus and human metapneumovirus (hMPV) using real-time reverse transcription-polymerase chain reaction (rRT-PCR), and adenovirus using real-time PCR. Although not all participating laboratories test for all viruses, all test for RSV. In RDS, de-duplication is carried out during the data importation process; samples taken from the same individual within a 6-week period are grouped as one record to capture a single episode of infection in an individual [16]. We included data from 13 of the 14 laboratories with consistent reporting of RSV results during the study period: Barts and The London, Birmingham, Bristol, Cambridge, Leeds, Leicester, Manchester, Newcastle, Nottingham, Royal Free Hospital, Southampton, Truro and the Reference Laboratory at PHE Colindale. Weekly data was extracted from calendar week 27 in 2010 to week 26 in 2014 for children aged $<5$ years. The extracted data include information on patient's date of birth, sex, date of sample, and whether the RSV laboratory test was positive for RSV, negative for RSV but positive for another respiratory virus, or negative for all viruses including RSV.

We compared the RDS study population to the national laboratory surveillance system, LabBase2, also held by PHE. LabBase2 is a long established laboratory surveillance system that covers England, Wales and Northern Ireland, but only includes records of positive RSV tests. Positive RSV test data from laboratories submitting to the RDS are also submitted to the national LabBase2 database. Several different types of laboratory tests are used for diagnosis of RSV infection by laboratories contributing to LabBase2, with the majority using genome or antigen detection methods. Similarly to the RDS, de-duplication is carried out during the data importation process; samples taken from the same individual within a 6-week period are assigned a unique identifier to capture a single episode of infection in an individual. We extracted data on all RSV-positive respiratory samples from LabBase 2 from children aged $<5$ years tested in all laboratories in England from week 27 in 2010 to week 26 in 2014, and only included the first sample of each episode of infection in this analysis.

\section{Statistical analysis}

The total number of RSV tests (positive and negative) in the RDS extract, the number of positive RSV tests in the RDS extract and the number of positive episodes in the LabBase 2 extract were summarized by age, month of birth, year [between week 27 (2010) and week 26 (2014)] and sex. We calculated the RSV positivity rate as the number of RSV-positive tests divided by the total number of RSV tests in the RDS extract by age, month of birth, sex and year. We defined RSV season onset as the first of two consecutive weeks in which the mean percentage of samples testing positive for RSV in the RDS was $\geqslant 10 \%$, and the end of RSV season as the last of two consecutive weeks in which the mean percentage of samples 
Table 1. Total number of tests (Respiratory DataMart System; RDS), number of respiratory syncytial virus $(R S V)$-positive tests (RDS), RSV positivity rate (RDS) and number of RSV-positive tests (LabBase2) in children aged $<5$ years from week 27 (2010) to week 26 (2014) by sex, age, birth month if tested for RSV in the first year of life, and year (week 27 to week 26)

\begin{tabular}{|c|c|c|c|c|}
\hline & \multicolumn{3}{|c|}{ Respiratory DataMart System } & \multirow{2}{*}{$\begin{array}{l}\text { LabBase } 2 \\
\text { RSV positive } \\
N(\%)\end{array}$} \\
\hline & $\begin{array}{l}\text { Total tests } \\
N(\%)\end{array}$ & $\begin{array}{l}\text { RSV positive } \\
N(\%)\end{array}$ & RSV positivity rate & \\
\hline Total & 63827 & 13034 & $20 \%$ & 30669 \\
\hline \multicolumn{5}{|l|}{ Sex } \\
\hline Male & $31278(49 \%)$ & $6165(47 \%)$ & $20 \%$ & $17050(56 \%)$ \\
\hline Female & $23577(37 \%)$ & $4848(37 \%)$ & $21 \%$ & $13332(43 \%)$ \\
\hline Unknown & $8970(14 \%)$ & $2021(16 \%)$ & $23 \%$ & $287(1 \%)$ \\
\hline Sex ratio $(M: F)$ & $1 \cdot 3: 1$ & $1 \cdot 3: 1$ & & $1 \cdot 3: 1$ \\
\hline \multicolumn{5}{|l|}{ Age } \\
\hline$<3$ months & $20467(32 \%)$ & $4982(38 \%)$ & $24 \%$ & $12641(41 \%)$ \\
\hline $3-5$ months & $9384(15 \%)$ & $2423(19 \%)$ & $26 \%$ & $6526(21 \%)$ \\
\hline 6-11 months & $11712(18 \%)$ & $2528(19 \%)$ & $22 \%$ & $6116(20 \%)$ \\
\hline 1 year & $10439(16 \%)$ & $1815(14 \%)$ & $17 \%$ & $3703(12 \%)$ \\
\hline 2 years & $4905(8 \%)$ & $670(5 \%)$ & $14 \%$ & $922(3 \%)$ \\
\hline 3 years & $3929(6 \%)$ & $402(3 \%)$ & $10 \%$ & $510(2 \%)$ \\
\hline 4 years & $2991(5 \%)$ & $214(2 \%)$ & $7 \%$ & $251(1 \%)$ \\
\hline \multicolumn{5}{|c|}{ Birth month (if $<1$ year old)* } \\
\hline January & $3271(8 \%)$ & $586(6 \%)$ & $18 \%$ & $1384(5 \%)$ \\
\hline February & $2774(7 \%)$ & $404(4 \%)$ & $15 \%$ & $994(4 \%)$ \\
\hline March & $2970(7 \%)$ & $436(4 \%)$ & $15 \%$ & $1066(4 \%)$ \\
\hline April & $2818(7 \%)$ & $448(5 \%)$ & $16 \%$ & $1132(4 \%)$ \\
\hline May & $3038(7 \%)$ & $525(5 \%)$ & $17 \%$ & $1344(5 \%)$ \\
\hline June & $3056(7 \%)$ & $646(7 \%)$ & $21 \%$ & $1519(6 \%)$ \\
\hline July & $3173(8 \%)$ & $700(7 \%)$ & $22 \%$ & $1906(8 \%)$ \\
\hline August & $3484(8 \%)$ & $906(9 \%)$ & $26 \%$ & $2484(10 \%)$ \\
\hline September & $3833(9 \%)$ & $1210(12 \%)$ & $32 \%$ & $3297(13 \%)$ \\
\hline October & $4626(11 \%)$ & $1593(16 \%)$ & $34 \%$ & $4088(16 \%)$ \\
\hline November & $4575(11 \%)$ & $1565(16 \%)$ & $34 \%$ & $3743(15 \%)$ \\
\hline December & $3945(9 \%)$ & $914(9 \%)$ & $23 \%$ & $2326(9 \%)$ \\
\hline \multicolumn{5}{|l|}{ Year } \\
\hline 2010-2011 & $19751(31 \%)$ & $4103(31 \%)$ & $21 \%$ & $8327(27 \%)$ \\
\hline $2011-2012$ & $14804(23 \%)$ & $2919(22 \%)$ & $20 \%$ & $7228(24 \%)$ \\
\hline $2012-2013$ & $15021(24 \%)$ & $3013(23 \%)$ & $20 \%$ & $7495(24 \%)$ \\
\hline 2013-2014 & $14251(22 \%)$ & $2999(23 \%)$ & $21 \%$ & $7619(25 \%)$ \\
\hline
\end{tabular}

* Percentage denominator is the total number in infants aged $<1$ year [i.e. total tests $(\mathrm{RDS})=41563$, RSV positive $(\mathrm{RDS})=$ 9933 and RSV positive $($ LabBase2) $=25283$ ].

testing positive for RSV was $\geqslant 10 \%$, a method which has been used in previous studies [17, 18].

We used multivariable logistic regression models to estimate the odds of a positive result (if tested for RSV) by birth month, using the RDS extract. Age group (0, 1, 2, 3, 4 years), sex (male, female, unknown) and year (2010-2011, 2011-2012, 2012-2013, 20132014) were investigated as potential confounders and were added to the model in a forward stepwise manner. We also included an interaction term between age group and birth month. We used likelihood ratio tests to determine whether the inclusion of a variable significantly improved the fit of the model; a likelihood ratio test $P$ value of $<0.05$ was considered significant. Robust standard errors were used to allow for clustering by laboratory. Infants born in January were used as the baseline group.

\section{RESULTS}

Characteristics of the study population are shown in Table 1. In the RDS there was an average of 15986 


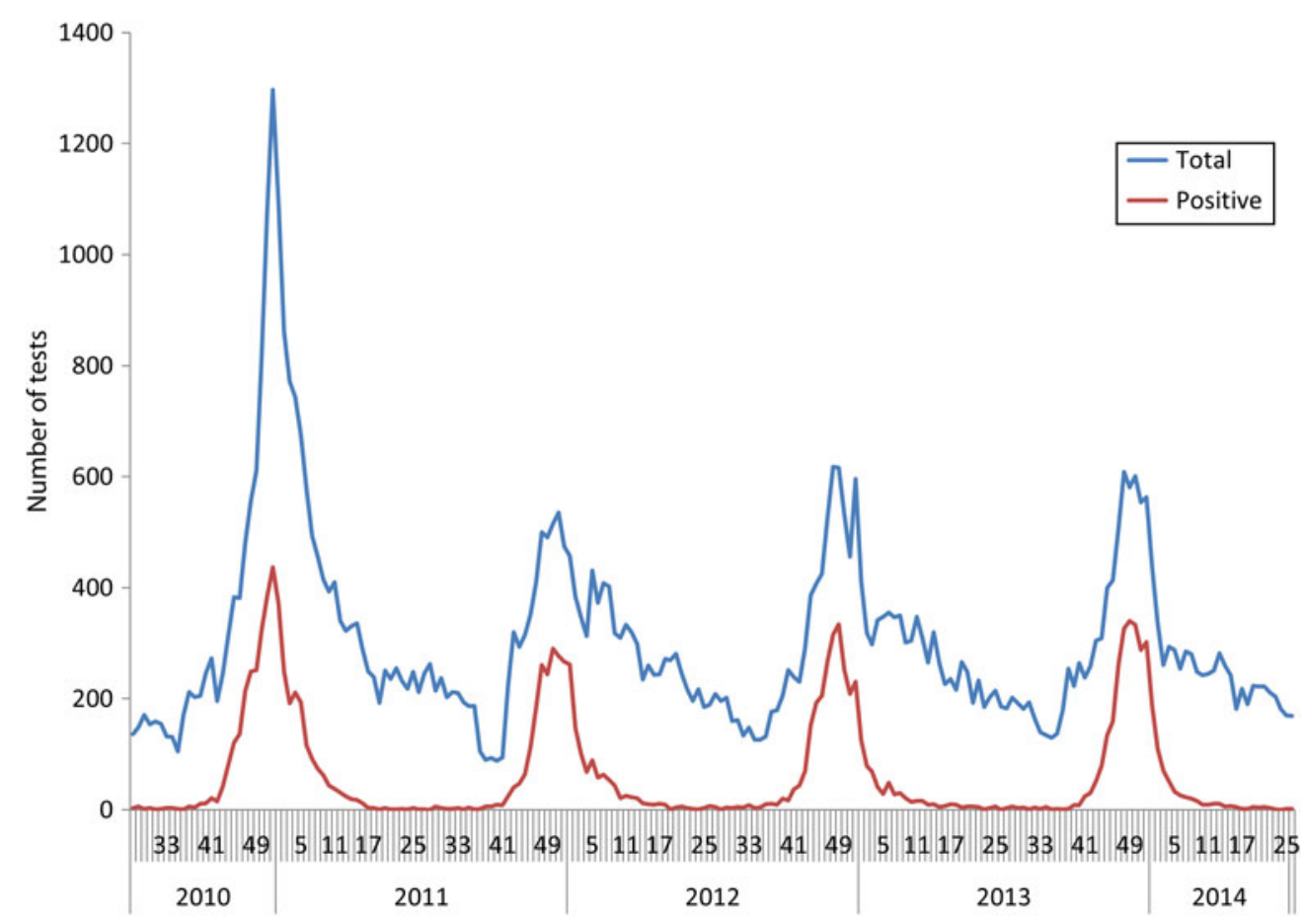

Fig. 1. Total number (blue) and number of positive (red) respiratory syncytial virus tests in children aged $<5$ years recorded in the Respiratory DataMart System from week 27 (2010) to week 26 (2014), over time.

tests and $3259 \mathrm{RSV}$ positives per year in children aged $<5$ years during the study period. Laboratory-confirmed RSV positivity showed a clear and consistent seasonal pattern; RSV season onset was in October each year (ranging from calendar weeks 41-43) during the study period and the end of RSV season ranged from January to March (weeks 4-10). Overall testing peaked during December in each RSV season of the 4 years studied (Fig. 1). The week with the highest proportion of positive RSV laboratory tests in children aged $<5$ years each season was week 48 in 2010-2011 (42\% positive), week 1 in 2011-2012 (55\% positive), and week 49 in 2012-2013 and 2013-2014 (52\% and 55\% positive, respectively). Of the 4 years included in the study, the highest number of RSV laboratory tests was carried out during the 2010-2011 RSV season. Of the RSV-positive tests in the RDS during the study period $(n=13034), 16 \%$ were also positive for at least one other respiratory virus. Of the RSV-negative tests in the RDS during the study period ( $n=50793), 41 \%$ were positive for at least one other respiratory virus.

The results by age, birth month and sex were very similar in the RDS and LabBase2 extracts (Table 1). The RDS results demonstrated that both the total number of tests and the number of positive tests decreased with increasing age (Fig. 2). Seventy-six per cent $(9933 / 13034)$ of RSV-positive tests in children aged $<5$ years over the study period were in infants aged $<1$ year, whereas only $2 \%(214 / 13034)$ were in in children aged 4 years. Moreover, $47 \%$ ( $29851 / 63827)$ of tests and $57 \%(7405 / 13034)$ of positives in children aged $<5$ years over the study period were in infants aged $<6$ months. The number of RSV positives peaked at age 1 month $(n=2198)$. Infants aged 1, 2 and 3 months had the highest rate of RSV positivity: 29\% (2198/7551), 29\% (1507/ $5194)$ and $27 \%(996 / 3697)$ tested positive for RSV, respectively. The highest number of tests was in infants aged $<1$ month $(n=7722)$. Infants aged $<1$ year who were born in September, October and November had the highest number and proportion of positive test results (Table 1). In LabBase2, 82\% (25 283/30 669) of positive results in children aged $<5$ years over the study period were in infants aged $<1$ year; only $1 \%$ of tests $(251 / 30669)$ were in children aged 4 years. The number of RSV-positive tests also peaked at age 1 month $(n=5326)$. Thirteen per cent $(3297 / 25$ 283) of infants with a positive RSV test in their first year of life recorded in LabBase2 were born in September, 16\% (4088/25 283) were born in October and $15 \%(3743 / 25283)$ were born in November. In both datasets the sex ratio (M:F) was 1·3:1.

The best fitting multivariable logistic regression model included sex, calendar year, age group and 


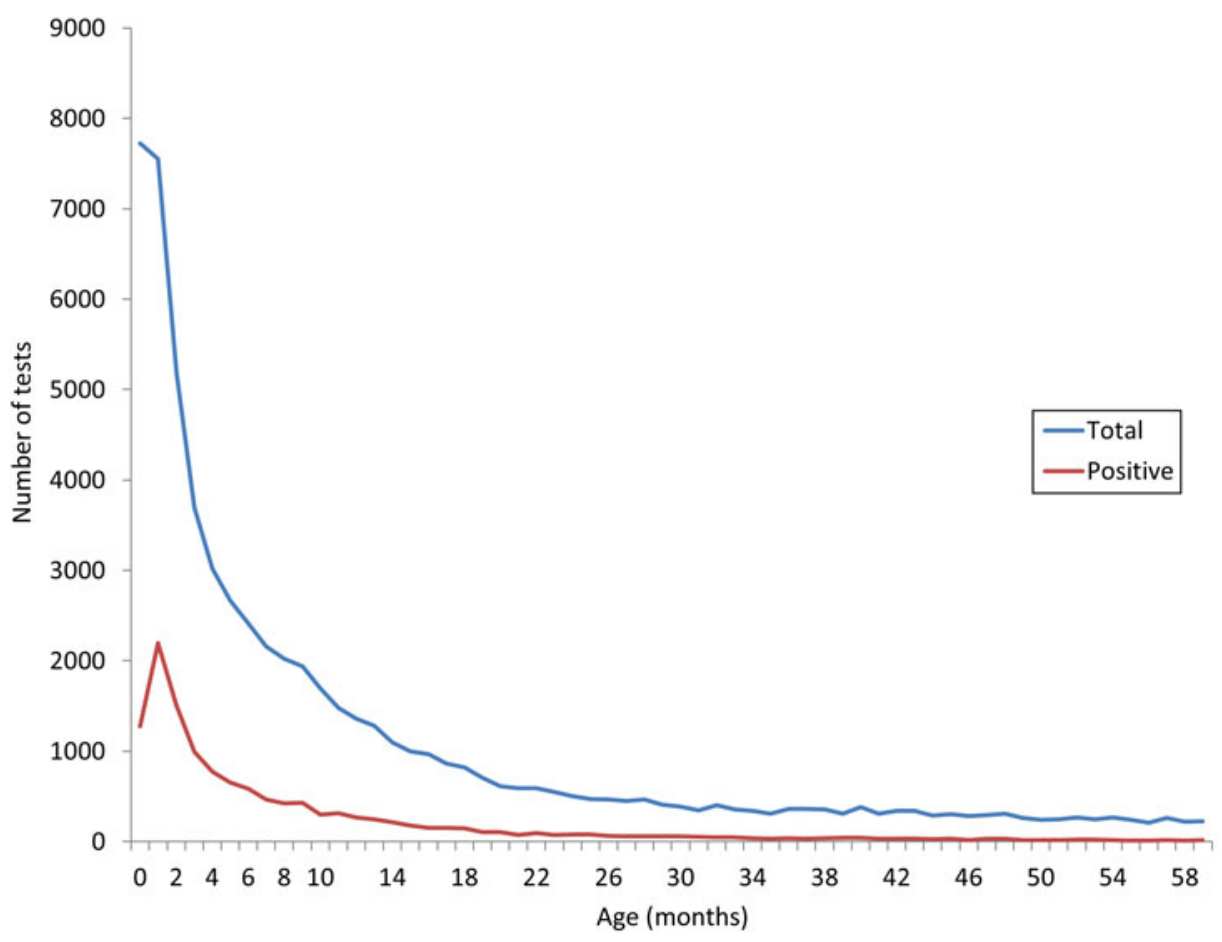

Fig. 2. Total number (red) and number of positive (green) respiratory syncytial virus tests in children aged $<5$ years recorded in the Respiratory DataMart System from week 27 (2010) to week 26 (2014), by age in months.

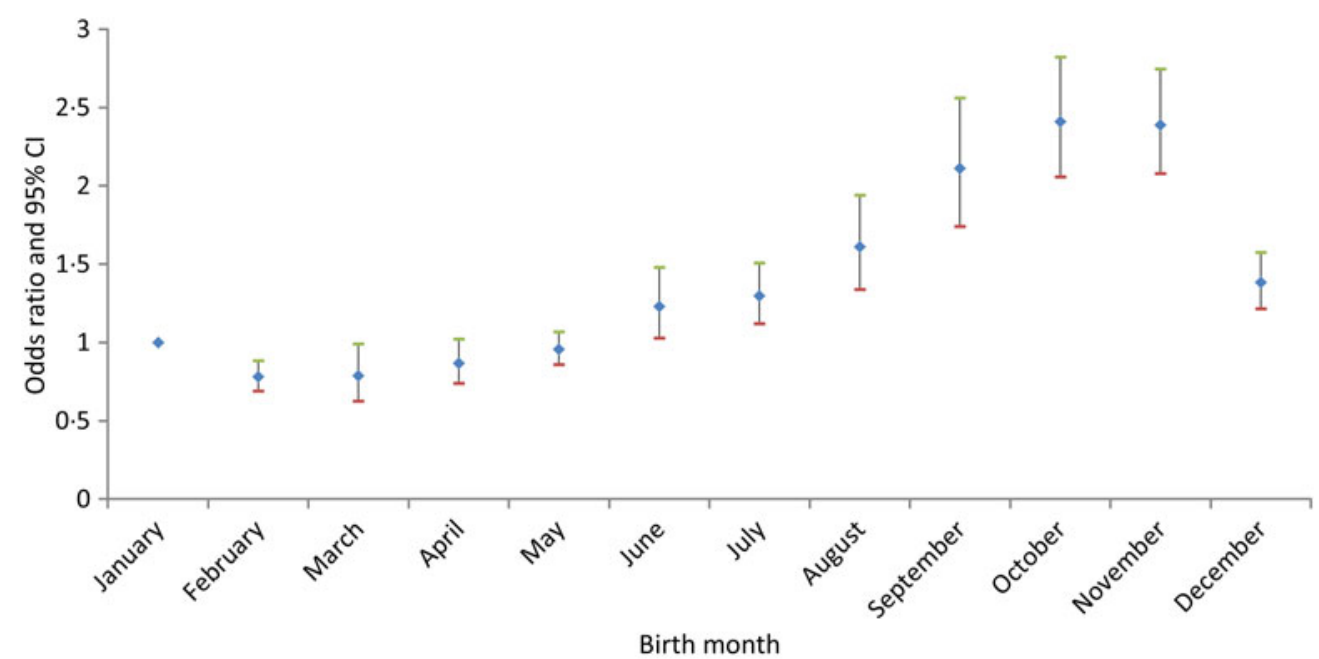

Fig. 3. Odds ratios and 95\% confidence intervals (CI) from final multiple logistic regression model using Respiratory DataMart System data to compare odds of a positive result if tested for respiratory syncytial virus by birth month, showing results for infants aged $<1$ year only. Infants born in January are the baseline group.

birth month as well as an age group $\times$ birth month interaction term. Infants aged $<1$ year born in September [odds ratio (OR) 2.1, 95\% confidence interval (CI) 1.7-2.7], October (OR 2.4, 95\% CI $2 \cdot 1-2 \cdot 8$ ) or November (OR $2 \cdot 4,95 \%$ CI $2 \cdot 1-2 \cdot 7$ ) had the highest odds of a positive result if tested for RSV in the first year of life compared to infants born in January (Fig. 3). The effect of birth month on odds of a RSV positive test result decreased with increasing age (Fig. 4). For example, infants aged 4 years born in September (OR 0.4, 95\% CI $0 \cdot 3-0 \cdot 6)$ and infants aged 4 years born in January (OR $0 \cdot 4,95 \%$ CI $0 \cdot 3-0 \cdot 6$ ) had the same odds of a positive result. 


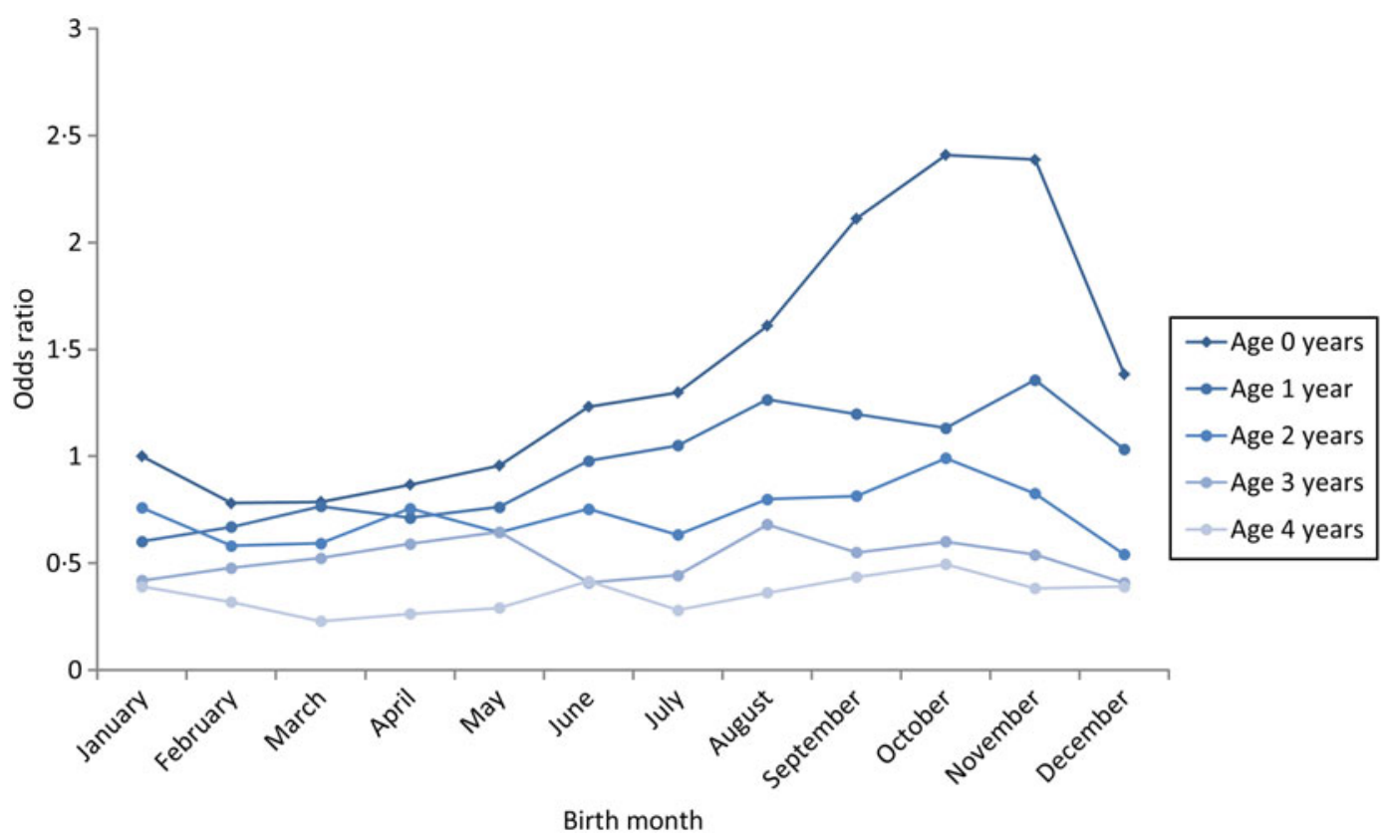

Fig. 4. Odds ratios from final multiple logistic regression model using Respiratory DataMart System data to compare odds of a positive result if tested for respiratory syncytial virus by birth month, stratified by age in years. Infants born in January are the baseline group.

\section{DISCUSSION}

This study shows that a significant proportion of laboratory-confirmed RSV infections in England recorded in two laboratory surveillance databases from 2010 to 2014 were in infants aged $<6$ months. In both datasets, there was a peak in RSV-positive tests in infants aged 1 month. RSV circulation was very consistent in timing each year, with a 3 -week range in season onset over the study period. Moreover, infants born near the beginning of an RSV season had significantly increased odds of a positive result if tested for RSV during the first year of life.

A strength of our study is the use of laboratoryconfirmed RSV infection rather than clinically diagnosed RSV. Using clinical diagnoses of RSV infection may include misclassification of diagnoses when laboratory tests were not carried out, as specific respiratory viral aetiologies cannot be differentiated clinically $[19,20]$. The use of clinical diagnoses alone may therefore lead to bias in associations between patient characteristics and RSV infection. However, a limitation of our study is that no clinical information on the individuals tested was available in either dataset. The majority of RSV records in the RDS and LabBase 2 are from hospitalized patients [16], and it is likely that only severe or complex cases requiring hospital admission will require laboratory confirmation of RSV infection as it would usually be unnecessary to investigate mild infection for the presence of RSV. However, without clinical information it is not possible to confirm whether or not this assumption is correct. In addition, most tests in both RDS and LabBase 2 were carried out on young children ( $<1$ year). It is therefore possible that differences in testing according to age means that RSV is less likely to be picked up in older children. Linkage between administrative hospital data and laboratory data would allow analysis of the potential association between clinical presentation, patient characteristics, the probability of being tested, and RSV positivity.

The considerable number of tests and RSV-positive results in infants aged $<6$ months and the peak in number and percentage of positive RSV tests in infants aged 1 month is consistent with existing literature that reports age $<6$ months as a significant risk factor for severe RSV infection [21] and a peak in RSV bronchiolitis at age 1 month [12, 22]. Young infants have been a high priority for vaccination due to the serious complications and subsequent morbidity that can occur following RSV infection in early life [6]. However, young infants are at risk of enhanced disease following vaccination as demonstrated during testing of the first candidate vaccine, formalin-inactivated RSV, in the 1960s [6]. The immaturity of the immune 
system and significant heterogeneity in the presence of maternal antibodies also present major challenges to vaccine development for this target group [23]. Nonetheless, a World Health Organization consultation in early 2015 on the development of RSV vaccines suggests it is likely that an RSV vaccine will be available commercially within 5-10 years [24], as major advances in the understanding of the biology of RSV and innovations in immunogen design have resulted in a number of promising potential vaccine candidates in clinical trials [25]. Our results highlight the importance of developing optimal strategies to prevent disease in young infants with these potential future vaccines.

Our analysis found RSV circulation to be highly consistent in timing each year, with only a 3-week range in season onset over the study period. The large peak in infants being tested for RSV during 2010-2011 can be attributed to the intense influenza season during this first post-pandemic winter period, as the RDS holds records of the results of samples tested simultaneously for multiple respiratory viruses including influenza and RSV [16, 26]. The large number of tests performed outside of the RSV season can also be attributed to testing for a range of respiratory viruses. The consistency between results from the RDS and LabBase2 suggest generalizability of the RDS results to the long established national surveillance system.

Our finding that birth in months SeptemberDecember was associated with increased odds of a positive result if tested for RSV in the first year of life in England supports the results of previous studies that show birth around the beginning of the RSV season is a risk factor for RSV-associated hospitalization [7-9, 11]. Several of these previous studies investigating month of birth as a risk factor for RSV-associated hospitalization were limited by sample size and all based outside of the UK. The largest study was carried out in the United States and suggests children born during December and January had a two- and threefold higher risk, respectively, of RSV-confirmed hospitalization during infancy than those born in July, although these cases were identified by International Classification of Diseases (Ninth Revision) codes only [7]. The increased risk of severe RSV infection in infants born close to the beginning of the RSV season is likely due to these infants having a longer exposure to RSV at a young age in combination with lower levels of maternal antibodies during the beginning of the RSV season and immaturity of the lungs [21]. The exact birth months with the highest risk of severe RSV infection in infancy varies between countries due to differences in the timing of RSV season, which highlights the importance of country-specific epidemiology studies including birth month as a potential risk factor when analysing severe RSV infection.

This study highlights the importance of young age ( $<6$ months) and birth near the beginning of the RSV season in risk of laboratory-confirmed RSV infection. Future vaccination programmes and other interventions should ensure protection for these groups is considered.

\section{DECLARATION OF INTEREST}

None.

\section{REFERENCES}

1. Bont L, et al. RSV - still more questions than answers. Pediatric Infectious Disease Journal 2014; 33:1177-1179.

2. Nair H, et al. Global burden of acute lower respiratory infections due to respiratory syncytial virus in young children: a systematic review and meta-analysis. Lancet 2010; 375: 1545-1555.

3. Welliver RC. Review of epidemiology and clinical risk factors for severe respiratory syncytial virus (RSV) infection. Journal of Pediatrics 2003; 143 (5 Suppl.): S112-117.

4. Hall CB, et al. Respiratory syncytial virus-associated hospitalizations among children less than 24 months of age. Pediatrics 2013; 132: e341-348.

5. Haynes LM. Progress and challenges in RSV prophylaxis and vaccine development. Journal of Infectious Diseases 2013; 208 (Suppl. 3): S177-183.

6. Anderson LJ. Respiratory syncytial virus vaccine development. Seminars in Immunology 2013; 25: 160-171.

7. Lloyd PC, et al. The effect of birth month on the risk of respiratory syncytial virus hospitalization in the first year of life in the United States. Pediatric Infectious Disease Journal 2014; 33: e135-140.

8. Houben ML, et al. Clinical prediction rule for RSV bronchiolitis in healthy newborns: prognostic birth cohort study. Pediatrics 2011; 127: 35-41.

9. Holberg CJ, et al. Risk factors for respiratory syncytial virus-associated lower respiratory illnesses in the first year of life. American Journal of Epidemiology 1991; 133: $1135-1151$.

10. Figueras-Aloy J, et al. FLIP-2 Study: risk factors linked to respiratory syncytial virus infection requiring hospitalization in premature infants born in Spain at a gestational age of 32 to 35 weeks. Pediatric Infectious Disease Journal 2008; 27: 788-793.

11. Cilla $\mathbf{G}$, et al. Risk factors for hospitalization due to respiratory syncytial virus infection among infants in the Basque Country, Spain. Epidemiology and Infection 2006; 134: 506-513.

12. Murray J, et al. Risk factors for hospital admission with RSV bronchiolitis in England: a population-based birth cohort study. PLOS ONE 2014; 9: e89186. 
13. Müller-Pebody B, et al. Contribution of RSV to bronchiolitis and pneumonia-associated hospitalizations in English children, April 1995-March 1998. Epidemiology and Infection 2002; 129: 99-106.

14. Pitman RJ, et al. Assessing the burden of influenza and other respiratory infections in England and Wales. Journal of Infection 2007; 54: 530-538.

15. Cromer D, et al. The burden of influenza in England by age and clinical risk group: a statistical analysis to inform vaccine policy. Journal of Infection 2014; 68: 363-371.

16. Zhao H, et al. A new laboratory-based surveillance system (Respiratory DataMart System) for influenza and other respiratory viruses in England: results and experience from 2009 to 2012. Eurosurveillance 2014; 19(3).

17. McGuiness CB, et al. Respiratory syncytial virus surveillance in the United States, 2007-2012: results from a national surveillance system. Pediatric Infectious Disease Journal 2014; 33: 589-594.

18. Mullins JA, et al. Substantial variability in community respiratory syncytial virus season timing. Pediatric Infectious Disease Journal 2003; 22: 857-862.

19. Thornton $\mathbf{H ~ V}$, et al. Clinical presentation and microbiological diagnosis in paediatric respiratory tract infection: a systematic review. British Journal of General Practice 2015; 65: e69-81.
20. Moore HC, et al. Use of data linkage to investigate the aetiology of acute lower respiratory infection hospitalisations in children. Journal of Paediatrics and Child Health 2012; 48: 520-528.

21. Sommer C. Risk factors for severe respiratory syncytial virus lower respiratory tract infection. Open Microbiology Journal 2011; 5: 144-154.

22. Alvarez AE, et al. Epidemiological and genetic characteristics associated with the severity of acute viral bronchiolitis by respiratory syncytial virus. Jornal de Pediatria (Rio de Janeiro) 2013; 89: 531-543.

23. Anderson LJ, et al. Strategic priorities for respiratory syncytial virus (RSV) vaccine development. Vaccine 2013; 31 (Suppl. 2): B209-215.

24. Modjarrad K, et al. WHO consultation on respiratory syncytial virus vaccine development report from a World Health Organization Meeting held on 23-24 March 2015. Vaccine 2016; 34: 190-197.

25. PATH. RSV vaccine snapshot - PATH Vaccine Resource Library, 2015 (http://www.path.org/vaccineresources/ details.php?i=1562). Accessed 18 January 2016.

26. Mytton OT, Rutter PD, Donaldson LJ. Influenza A (H1N1)pdm09 in England, 2009 to 2011: a greater burden of severe illness in the year after the pandemic than in the pandemic year. European Centre for Disease Prevention and Control (ECDC) - Health Comunication Unit, 2012. 